\title{
EPHEMERAL NARRATIVE SYSTEMS' SOCIABILITY MEDIATED BY USER EXPERIENCE: A CASE STUDY OF INSTAGRAM STORIES
}

Thiago Alexandre de Souza Silva, Glívia Angélica Rodrigues Barbosa, Ismael Santana Silva, and Flavio Roberto dos Santos Coutinho Centro Federal de Educação Tecnológica de Minas Gerais, Brazil

\begin{abstract}
The use of ephemeral media platforms, like Instagram Stories, has been growing and the ephemeral characteristic of these platforms has created a new form of interaction mediated by technology. This work aimed to characterize how Instagram Stories supports the sociability among its members and to discuss the impact of this proposed sociability in the social interaction of users. We conducted this research in three steps. First, we executed an evaluation in a perspective of Human-Computer Interaction (HCI) experts, which aimed to analyze whether the features offered by Instagram Stories are adequate to user experience (UX) and sociability guidelines. Next, we performed an evaluation from the users' perspective, in order to investigate users' experience while they are interacting through Instagram Stories. Finally, we made a triangulation of the results and we discussed the impact of Instagram Stories in the social interaction of users. The results showed that the interface and interaction model of Instagram Stories reflects directly on the experience of use and sociability of its members, once this system offers features to support the social interaction, but the way these features are available on interface may limit the sociability among its users. As contributions, this work reinforces the importance of sociability as a strategy of use quality to improve user experience and social interaction on collaborative systems. Besides that, we present recommendations and insights to Collaborative Systems and HCI communities about the design and evaluation of ephemeral communication services.
\end{abstract}

\section{KEYWORDS}

Online Social Networks, Ephemeral Communication, Sociability, User Experience, Interface Evaluation, Instagram Stories 


\section{INTRODUCTION}

Ephemeral communication platforms (e.g., Instagram Stories, Whatsapp Status and Facebook Stories) have become increasingly popular (Xu et al, 2016). Through this kind of service, users can share contents (e.g., text, images or videos), which are exhibited temporarily on the system. In other words, the shared content has an exhibit deadline on ephemeral media platforms (Cavalcanti et al, 2017).

The ephemeral characteristic of these platforms has created a new form of social interaction mediated by technology. On this environment, users are encouraged to interact continuously to visualize and share new contents with their friends. This continuous interaction is necessary because the shared content is available just for a short time. For instance, a content shared on Whatsapp Status and Instagram Stories are available only for 24 hours (Bayer et al, 2016) (Xu et al, 2016) (Cavalcanti et al, 2017).

Considering this scenario, ephemeral communication services bring challenges to Collaborative Systems community and Human-Computer Interaction (HCI) community concerning the user experience (UX) and sociability. Once it is necessary to maximize the satisfactory experience of use and to continuously stimulate the quality of social interaction (i.e., sociability (Preece, 2000) (De Souza \& Preece, 2004)) on these kind of system (Bayer et al, 2016) (Cavalcanti et al, 2017).

In this context, an investigation line which has started to be explored refers to the characterization of the interaction phenomena concerning the usage of the ephemeral communication systems. In literature there are some works (e.g., Cavalcanti et al. (Cavalcanti et al, 2017) and Xu et al. (Xu et al, 2016)) which discussed how users are reacting to Snapchat's ephemerality proposal. However, researchers as Cavalcanti et al. (Cavalcanti et al, 2017) and $\mathrm{Xu}$ et al. (Xu et al, 2016) explain that it is necessary to conduce more researches in this line to better understand how ephemeral communication platforms are impacting on the social interaction of the users. From this kind of investigation it could be possible to generate insights and recommendations to the design and evaluation of ephemeral communication systems more focused in UX and sociability (De Souza \& Preece, 2004) (Wilson et al, 2009) (Preece et al, 2015) (Bayer et al, 2016) (Xu et al, 2016) (Cavalcanti et al, 2017).

Given the importance of exploring this research line, the main goal of this work is to evaluate and to characterize the sociability on ephemeral narratives platforms. In order to achieve this goal, we conducted a case study on Instagram Stories (Instagram, 2016). On this platform, the ephemeral contents are called "Stories". To share their stories, the users may publish different kinds of temporary content (e.g., images, videos and texts). Each shared content (i.e., story) has an exhibition time and it is available for up to 24 hours. The social interaction on Instagram Stories takes place through the visualization and interaction with the story shared by the users. For instance, when a user visualize an ephemeral content, he may react or comment the publication as well as send a message to the owner of the shared content (Instagram, 2016) (Xu et al, 2016) (Bayer et al, 2016). We chose to perform a case study on Instagram Stories to explore our research question, once it is one of the most used platforms to ephemeral communication in worldwide (Instagram, 2016) (Statista, 2018).

Considering the aim of this work, we investigated the following research question: "Does the interface and interaction model of Instagram Stories supports the social interaction among its users?". The adopted methodology to answer this question consisted in three steps. Initially, we performed an evaluation in the perspective of HCI experts to analyze whether the features 
offered by Instagram Stories are adequate to UX guidelines (Nielsen \& Budiu, 2015) (Neiva et $\mathrm{al}, 2016)$ and also sociability guidelines (De Souza \& Preece, 2004) available in literature. Later, we conducted an evaluation in the users' perspective, which aimed to analyze use experience during the social interaction mediated by this system. Finally, we triangulated the evaluations' results and we discussed the impact of Instagram Stories in the social interaction of its members.

The main results indicated that the interface and interaction model of Instagram Stories offers features to support the social interaction, but the way these features are available on interface may limit the sociability among its users. Thus, this research contributes to Collaborative Systems community and HCI community may reflect about the importance of addressing the principles of UX and sociability in their ephemeral narratives systems. This work also reinforces the importance of sociability as a strategy of use quality to improve user experience and social interaction on collaborative systems. In addition, the proposed methodology may be reproduced in the evaluation of other ephemeral narratives platforms, considering the UX and social interaction of different interest groups (e.g., children, people with disabilities). Therefore, our findings provide insights that are useful for designing of ephemeral communication systems more sociability-focused in the future.

\section{RELATED WORKS}

In literature, there are some works (e.g., Cavalcanti et al. (Cavalcanti et al, 2017) and Xu et al. (Xu et al, 2016)) which explore the interaction phenomena concerning the use of the ephemeral communication systems. In this research line, Bayer (Bayer et al, 2016) discussed the influence of ephemeral narratives systems on users' interaction. To achieve the research goal, Bayer (Bayer et al, 2016) investigated how the ephemeral narratives (e.g., temporary images and videos) shared on Snapchat has been consumed by the users. As contribution, Bayer (Bayer et al, 2016) showed dimensions which characterize the ephemeral interaction among users of Snapchat.

Cavalcanti et al. (Cavalcanti et al, 2017) discussed the strategies created by users to save the media, the meaning and the context of shared ephemeral content. Cavalcanti et al. (Cavalcanti et al, 2017) conducted an interview with fifteen Snapchat users and their results showed that the participants developed alternative actions to avoid the loss of ephemeral content, such as to take screenshots of shared contents by friends or to save their self-publication. As contribution, Cavalcanti et al. (Cavalcanti et al, 2017) present reflections regarding to the purpose of ephemeral narratives and the way that users are using these services.

Xu et al. (Xu et al, 2016) investigated how Snapchat supports the interaction of its users with ephemeral contents. Xu et al. (Xu et al, 2016) interviewed twenty-five Snapchat users to characterize their experiences and perceptions during the use of Snapchat. As results and contributions, this research presents the possible impacts of the interface model of this kind of system in the users' experience.

Although different interaction phenomena related to ephemeral communication systems have been explored, we did not find works which investigate how this kind of system is impacting on the quality of sociability among their users. Therefore, our study directly complements the prior works and we also contribute to future works in this research line. In our analysis regarding to the interaction phenomena on ephemeral narrative platforms, we focused in the perspective of impact of these systems in social interaction of their users. 
Furthermore, our research contributes to Collaborative Systems community and HCI community may reflect about the importance of addressing UX and sociability in their ephemeral narratives systems.

\section{SOCIABILITY ON INSTAGRAM STOIES THROUGH EXPERTS' PERSPECTIVE}

In the first step of our research, we performed an evaluation in the perspective of HCI experts to analyze whether the features offered by Instagram Stories are adequate to UX guidelines (Nielsen \& Budiu, 2015)(Neiva et al, 2016) and also sociability guidelines (De Souza $\&$ Preece, 2004) available in literature. Next, we present the method and the main results of this step.

\subsection{Method}

We performed the proposed evaluation using the Heuristic Evaluation (HE). HE is an inspection method conducted by experts in HCI to evaluate the interface and interaction model of a system. Through this method, the expert judges the compliance of the interface concerning a set of guidelines (also called of heuristics) (Nielsen, 1994). For each potential problem identified on interface (i.e., violation of guideline), the evaluator associates one or more infringed guidelines and assigns a degree of severity. The severity scale ranges from 0 to 4 , where the higher the value, the more serious the impact of the problem in the user interaction (Nielsen, 1994).

In addition to the HE method, Nielsen (Nielsen, 1994) also presented a set of 10 usability heuristics that could guide the evaluators during the inspection. However, Nielsen (Nielsen, 1994) also emphasized that the set of guidelines used to guide the HE could be adapted according to the purpose of the inspection. In other words, the evaluators could evaluate the interface following the steps of the $\mathrm{HE}$ guided by other guidelines of use quality (e.g., accessibility, communicability, UX and sociability) (Nielsen, 1994)(Nielsen \& Budiu, 2015).

We performed a HE guided by the UX guidelines presented by Nielsen and Budiu (Nielsen $\&$ Budiu, 2015) and Neiva et al. (Neiva et al, 2016) and the sociability guidelines presented by de Souza and Preece (De Souza \& Preece, 2004). We adopted these guidelines once the user experience impacts in the quality of social interaction (i.e., sociability) mediated by systems (De Souza \& Preece, 2004)(Wilson et al, 2009)(Preece et al, 2015)(Nielsen \& Budiu, 2015). Thus, it is important to inspect and to characterize whether the features of Instagram Stories are adequate to support the social interaction of its members.

The UX guidelines (Nielsen \& Budiu, 2015) (Neiva et al, 2016) used were: UX1 - Contextualization to the user and adequacy to the features; UX2 - Error handling and user's assistance; UX3 - Clear, immediate and informative feedback; UX4 - Explain interaction paths; UX5 - Adaptable; UX6 - Perceptible; UX7 - Responsive design; UX8 - Navigable; UX9 - Readable; UX10 - Predictability and consistency; UX11 - Visibility and easy access to all existing information; UX12 - Minimization of user's memory load; UX13 - Easy access to the features; UX14 - Help and documentation; UX15 - Good use of screen space; and UX16 - Encouraging interaction. 
The sociability guidelines (De Souza \& Preece, 2004) used were: S1 - Allow people to develop their own identities online; S2 - Encourage empathy and trust among members; S3 - Encourage reciprocity; S4 - Keep discussions in evidence and in topics; S5 - Maintain different options of communication and interaction; and S6 - Guarantee protection and privacy.

We conducted this evaluation on the first half of October 2017 with three evaluators with experience in applying HE. During the evaluation the experts considered eight distinct interaction scenarios on Instagram Stories: (1) Share a story, (2) Add face filter, markers (people and location), emojis and polls; (3) Post videos with different effects; (4) Save stories on the device; (5) Interact with stories shared by friends; (6) Comment other stories; and (7) Delete an already published story.

After the inspection steps, the collected data by three experts were analyzed and consolidated in order to demonstrate the compliance of ephemeral communication features offered by Instagram Stories to the guidelines of UX and sociability. Later, the consolidated results were validated by an expert in HCI (i.e., a professional with more than seven years of experience). The following section summarizes the main results achieved in this step.

\subsection{Results}

Through the evaluation, we found a total of 12 potential problems that violated at least one UX or sociability guideline. Furthermore, $50 \%$ of the problems were classified with severity 4 (i.e., catastrophic problems), 33\% with severity 3 (i.e., big problems) and $17 \%$ with severity 2 (i.e., minor problems).

Among the identified problems, we highlight one which is related to the actions to navigate between the published stories (e.g., to return, to stop or to see the next story). During the visualization of shared content in sequence, we observed that it may be hard to the users to identify how to stop or to return to the exhibition of a specific story. This potential problem, showed on figure 1, may occur because the Instagram Stories' interface does not make the options clear to this kind of navigation.

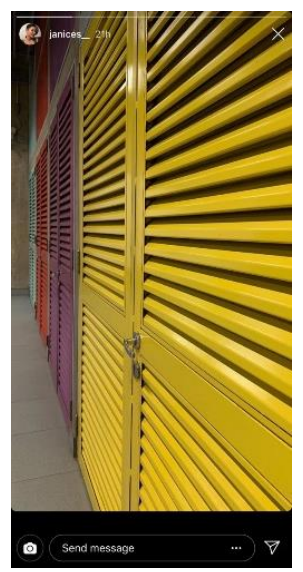

Figure 1. Example of a Potential Problem Found during Story's Visualization 
Another problem found is related to ambiguous interpretation between some interface elements while the user is editing a story. As the figure 2 shows, the interface displays two signs in different moments during story making that have almost the same smiling face and design. One is used to apply face filters and the other to add emojis, markers and gifs. However, that was considered as a problem since the user may not distinguish one's functionality from the other's, causing confusion.

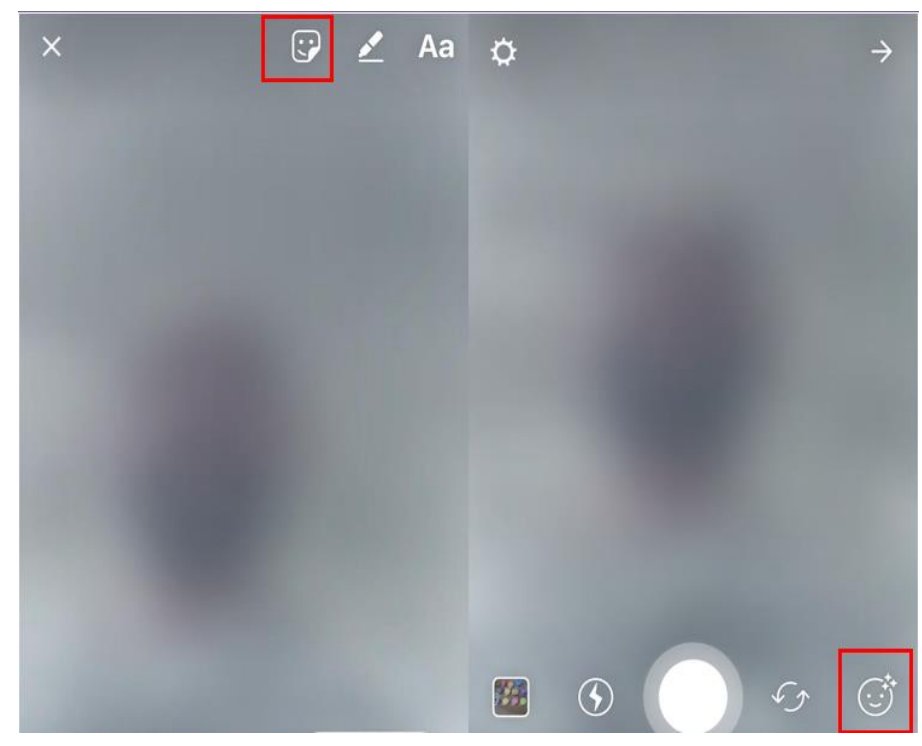

Figure 2. Example of a Potential Problem Found during Story's Edition

We also found a potential problem concerning accessing markers (location or user profile) on published stories. Since Instagram Stories' interface values maximum screen usage, depending on where users placed a marker, it is difficult to access it if the marker is on the same space where the comment bar is placed. This situation, made explicit on figure 3 , leads to errors in screen usage, because it is preventing the user from interacting with this type of markup.

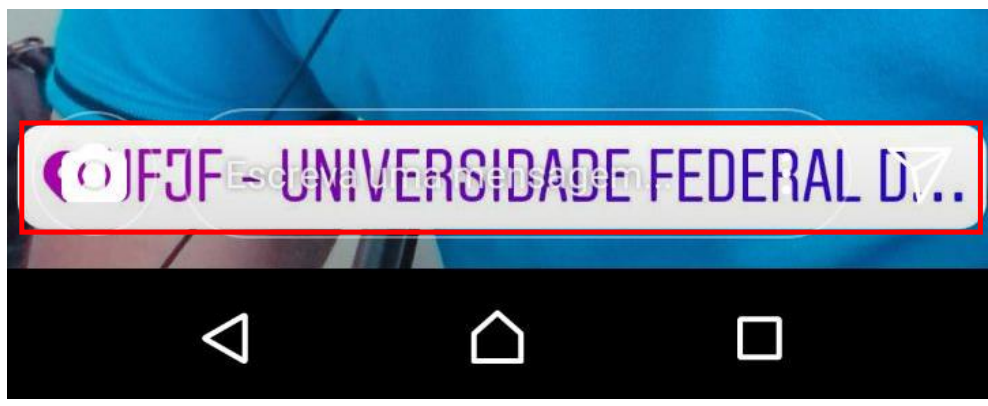

Figure 3. Example of a Potential Problem Found during Story's Visualization 
Since each potential problem may be associated with the absence of one or more UX and sociability guidelines and the same guideline may be violated by different problems, we checked the frequency that each guideline was violated in the evaluation, such as shown in Figure 4.

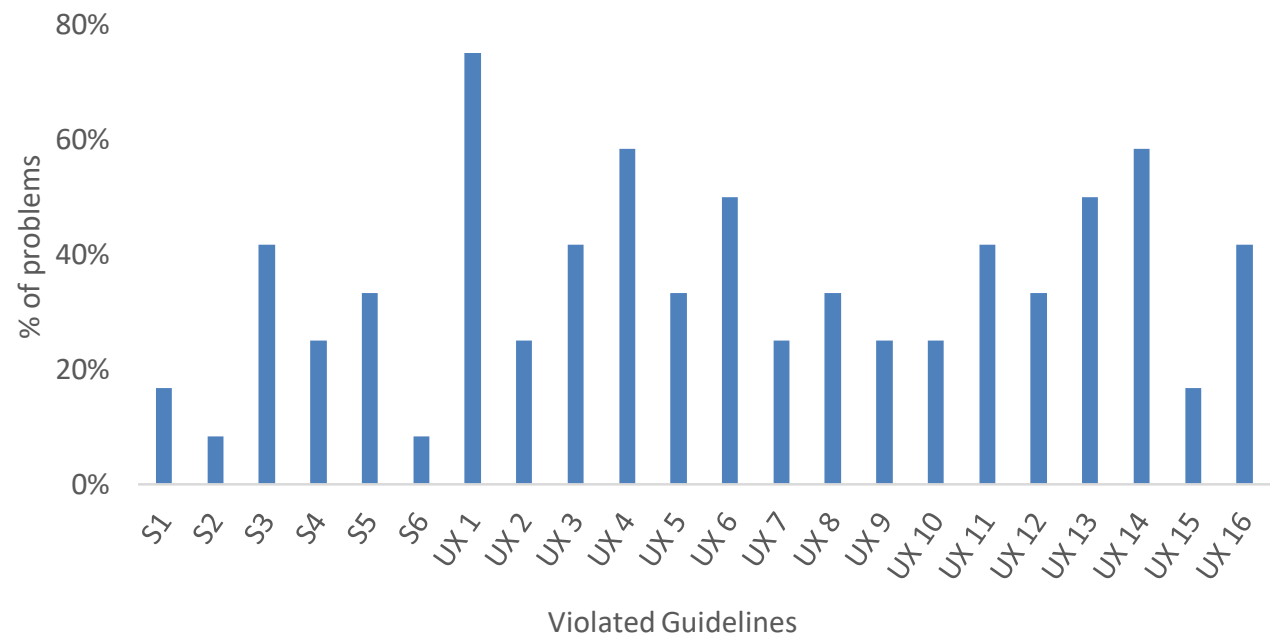

Figure 4. Violation Frequency of UX and Sociability Guidelines on Instagram Stories

From the data presented in Figure 4, we observed that guidelines "UX1 - Contextualization to the user and adequacy to the features", "UX4 - Explicit paths of interaction", "UX6 - Perceptible", "UX13 - Easy access to features" and "UX14 - Help and documentation" were violated in at least $50 \%$ of the problems. In turn, the guidelines such as "S3 - Encouraging reciprocity", "S5 - Maintain different options of communication and interaction", "UX3 - Clear, immediate and informative feedback", "UX12 - Minimization of user's memory load" and "UX16 - Encouraging interaction" were violated in at least 35\% of the identified problems. Besides that, most of these potential problems were classified as big or catastrophic.

The violation frequency of these guidelines may be explained by an intrinsic relationship among them. Whether the system does not use elements in its interface model to contextualize the user about the possibilities of use and social interaction, the users may not perceive or access the offered mechanisms (Nielsen \& Budiu, 2015) (Bayer et al, 2016). Consequently, the Instagram Stories' users may not interact with other members through the resources to ephemeral communication (De Souza \& Preece, 2004) (Preece et al, 2015) (Nielsen \& Budiu, 2015) (Bayer et al, 2016).

In summary, we observed that the interface and interaction model of Instagram Stories presents potential problems that may limit the experience of use as well as the social interaction mediated by ephemeral communication. These results suggest that the interface and interaction model of Instagram Stories can be improved to support the sociability of its members. 


\section{SOCIABILITY ON INSTAGRAM STORIES THROUGH USERS' PERSPECTIVE}

In order to evaluate and to characterize the experience of use and sociability on Instagram Stories from the perspective of its members, we performed two different evaluations. The first one was an evaluation with users in a controlled environment (Preece et al, 2015) (Hassenzahl, 2013). From this approach, we analyzed the impacts of the problems reported by HCI experts in the social interaction of users from Instagram Stories. Later, we applied an online survey and the users' answers were analyzed in order to characterize their perceptions and opinions concerning the sociability proposal of Instagram Stories.

\subsection{Evaluation in Controlled Environment}

We performed the evaluation in controlled environment in three stages: preparation, execution and analysis. All stages were conducted by two evaluators (Hassenzahl, 2013). During the preparation stage, we determined the aims and the tasks to be performed during the evaluation. Furthermore, we defined the participants' profile as well as consent term. Finally, before the execution stage, we conducted a pilot test in order to verify whether all definitions and generated artefacts were adequate for the evaluation (Hassenzahl, 2013).

In the execution stage, we performed the evaluations with each selected participant. During this stage, for each evaluation session, we followed the following steps: (1) user's reception, (2) presentation of the goals of the evaluation, (3) take the formal consent of the participant,

(4) applying the pre-test questionnaire, (5) observation of the tasks performed by the user and (6) interview after test (Hassenzahl, 2013).

We proposed the following tasks to the participants: (T1) Post a photo or video captured by the application; (T2) Post a selected photo or video from the album on participant's device; (T3) Mark a friend in a photo or video in the published story; (T4) Mark a location in a photo or video in the published story; (T5) Create a poll in a story; (T6) Post a video with boomerang effect; (T7) Post a video with emojis; (T8) Post a photo or video on the story with facial filter; (T9) Perform a live; (T10) View friends stories; (T11) Access bookmarks in friend's stories; (T12) Comment on a story; (T13) Save a story; and (T14) Delete a published story.

During each session of evaluation, the evaluator observed and registered: (1) the level of completion of each task (i.e., completed without error, completed with error or not completed) and (2) questions and observations made by the participant at the end of the test during the final interview.

We conducted a total of 25 sessions of evaluation with different participants. The 25 participants access Instagram Stories at least three times per week and they have been using this service for at least one year. Regarding the age, $72 \%$ of the participants were in the age group between 20 to $29,12 \%$ of them were in the group under the 20 years old and $16 \%$ of the participants were in the group over 29 years old. Further, $55 \%$ of the participants were female and $45 \%$ male. These 25 participants were selected by data saturation (Marshall, 1996).

After the sessions of evaluation, we analyzed the obtained data in order to verify: (1) the completion rate of each task, (2) the difficulties reported during the evaluations and (3) the possible impact of all these factors on the experience and socialization of users during the interaction mediated by Instagram Stories. 


\subsubsection{Results and Discussions}

Regarding the execution of the tasks, the graph in Figure 5 shows the completion percentage of each task by the participants. From these data, it is possible to observe that eight tasks (57\%) were completed with error or have not been completed for at least nine users. Furthermore, only four tasks (28\%) were completed (with or without errors) by all participants.

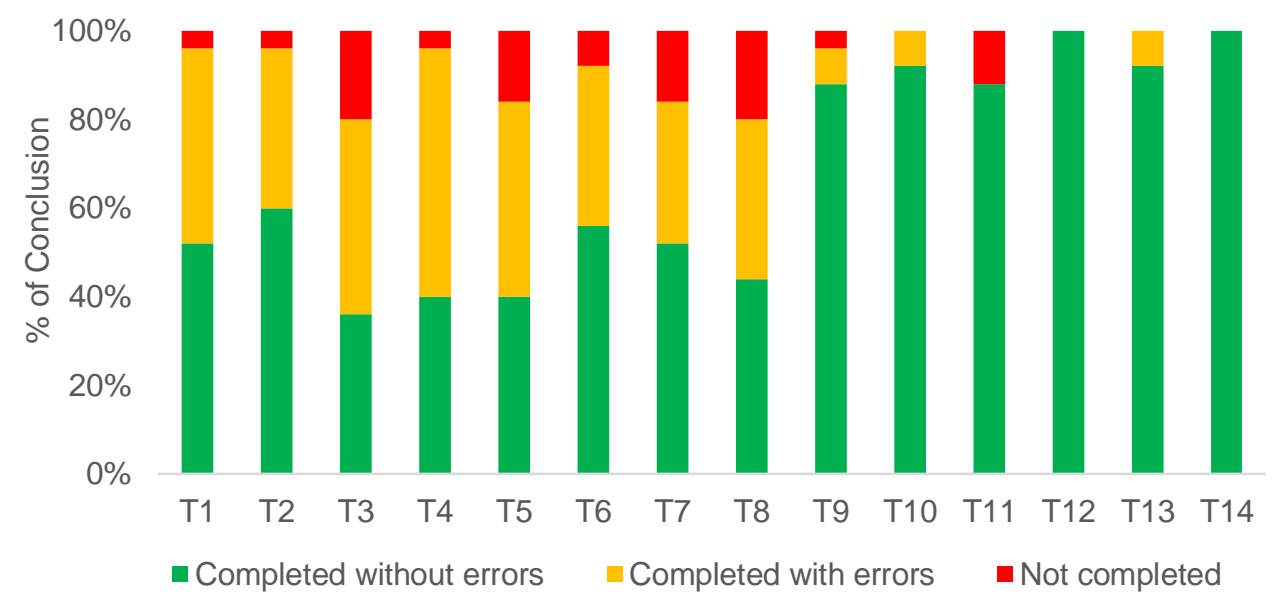

Figure 5. Percentage of Completion of Tasks by Users

To better understand these results, we asked to the participants about their experiences during the tasks execution. Analyzing the tasks individually, we observed that, during execution of tasks "T1 - Post a photo or video captured by the application" and "T2 - Post a selected photo or video from the album on participant's device", the participants used a different interaction path to post stories. As it shows in figure 6, there are two interface elements responsible to post the story. The sign "Your Story" posts the story directly in user stories' feed, while the "Send to" sign leads the user to another screen and then the user can share the story. For participants choosing to follow this extended interaction path to publish a story, it denotes that there is a potential problem, since there is a specific interface element to execute this action. That situation was observed by the evaluators throughout the execution of T1 to T8.

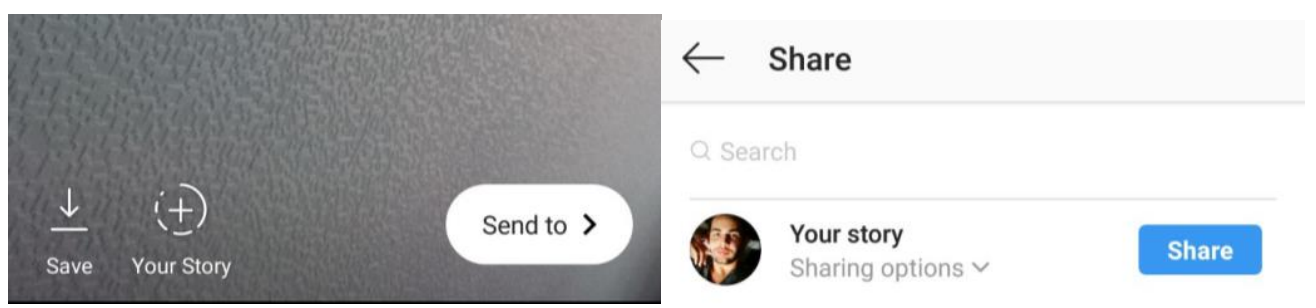

Figure 6. Instagram Story's Interface for Posting Stories

Regarding the tasks "T3 - Mark a friend in a photo or video in the published story" and "T4 - Mark a location in a photo or video in the published story", most users justified the errors by not knowing how to perform the profile markup in a story on Instagram Stories' interface. 
One user said: "First, I published the story without the markup, because I did not know this system feature. When you asked me to do it, I did not find the option on interface. So, I sent the story directly to the person who I wanted to mark" [P15]. Other participant reported: "I had problems in this task because the elements on interface of Instagram Stories are not compatible with my expectations and experience of interaction. I think that Instagram should adjust its interface to improve my interaction with my friends from the stories" [P07].

Regarding the task "T5 - Create a poll in a story", most participants reported problems related to (1) feedback, (2) difficulty of perception and access to the features and (3) social interaction. For instance, one participant described his negative experience during the task: "I would like to create a poll to propose a new way to interact with my friends from my publication. But, when I tried to do this task, I gave up. It was so hard to me, because I could not understand how to do this" [P03]. Another participant said: "I did not do it, because I could not identify on the interface elements referring to polls. So, I just interact with my friends from comments on the published stories" [P23].

After executing task "T6 - Post a video with boomerang effect", some participants had, not only, the same problems reported in T5, but also had problems with Instagram. The app malfunctioned while the user was creating the story and lost all the story production. This is a problem because Instagram does not display a clear message about the error and does not offer a possibility to recover the story, and then, able the user to continue editing it and publish it.

Taking in consideration tasks "T7 - Post a video with emojis" and "T8 - Post a photo or video on the story with facial filter", the participants reported difficulties since the interface does not make it clear in which interface element the functionality was located. It was also reported that: "The interface element where I can put face filters is almost the same to the element to add emojis and markers. So, I did not know, at first, what was the moment to use it, if it was before or after the picture was taken for the story" [P04]. Another user that participated in the evaluation reported that: "It is frustrating when you want to post something funny on Instagram Stories and you have to search around all the interface to find where the facial filters are. It took me a long time to find out where the element interface was and when I have to apply the filters" [P20].

Regarding the tasks "T9 - Perform a live" and "T13 - Save a story", user that completed with errors or did not concluded the tasks faced the same obstacles during execution of the tasks and interaction with the system. These users argued that they (1) did not know how to perform the activity asked, because they did not find an interaction and interface element that induced them to it, and (2) malfunction of the application, making the user restart the application several times to continue the task.

Users that faced difficulties completing task "T10 - View friends' stories" reported that the Instagram Stories' interface does not explicit the commands to go forward and backwards, or pause the story exhibition in addition, many users discovered this command by surprise, after interacting with the interface. By not making clear the options to user's interaction, the user experience could be compromised or limited.

For task "T11 - Access bookmarks in friend's stories", despite the majority (88\%) concluded without errors, the participants that faced problems and did not completed the tasks reported that the location or profile markup, required on T3 and T4, were positioned on places that it was difficult to access or the interface interpreted to a command to go forward or backwards on the story's list. A participant stated that: "I got really angry, because I could not access the location of my friend's story. This could help me to hang out with her at that place, but the Instagram Stories interprets that I want to go to the next story, and I do not" [P12]. 
Finally, tasks "T12 - Comment on a story" and "T14 - Delete a published story" were completed without errors by all the participants. Some of them reported that executing previous tasks lead them to find elements on the interface that could execute what the tasks required and, in botch tasks, did not take them a long time.

From the collected data and the experiences reported by the participants on this step of our research, we observed that the problems reported by HCI experts (in the previous step), indeed, have been impacting in the sociability of Instagram Stories' users. By violating UX and sociability guidelines related to (1) ease of use, (2) feedback, (3) flexibility and (4) stimulus of social interaction, Instagram Stories does not use interface and interaction elements consistent with the expectation and experience of participants. Thus, these users faced difficulties to recognize and to use all offered features to socialization from ephemeral communication (De Souza \& Preece, 2004) (Preece et al, 2015) (Nielsen \& Budiu, 2015) (Bayer et al, 2016).

\subsection{Sociability Survey}

After observing users' experience during social interaction on Instagram Stories in an evaluation in controlled environment, we analyzed the users' perceptions and opinions concerning to the offered features to stimulate social interaction on this ephemeral communication platform. To achieve this goal, we applied an online survey, which had nine questions. We grouped the questions so that it was possible to identify: (1) the participants' profiles and, (2) the participants' perceptions about the sociability resources of Instagram Stories and (3) suggestions for improvements to enhance the experience of use and sociability on this platform.

The survey took place from December 2017 to February 2018, with 225 users of Instagram Stories of Brazil. We focus on Brazilian users since Brazil figures among the top- 3 countries on the number of social media users (comScore, 2015). The survey was conducted and analyzed by three authors of this study.

\subsubsection{Profile of Participants}

The 225 participants access Instagram Stories at least three times per week and they have been using these services for at least one year. Regarding the age, $58 \%$ of the participants were in the age group between 20 to $29,19 \%$ of them were in the group under the 20 years old and $23 \%$ of the participants were in the group over 29 years old. Furthermore, $51 \%$ of the participants were female and $49 \%$ male.

\subsubsection{Users' Perceptions and Suggestions about the Sociability Features}

Initially, participants were asked to answer how many friends they kept on Instagram Stories. Most of the participants (67\%) had more than 500 friends on Instagram Stories and 33\% had between 200 and 500 contacts in this platform of ephemeral narratives. In addition, we asked the participants about their motivations for using Instagram Stories. All participants reported that they are using this platform, mainly, to share their great moments with friends and to see how these friends react to this shared content.

For instance, one participant said: "I'm using Instagram Stories because I like to share my photos and to see who will express some reaction regarding them before the expiration time" [P231]. Another participant reported that, even he does not know all features of Stories, he uses it to interact with the ephemeral content shared by his friends [P001]. Furthermore, to 83\% of participants, the fact of this shared content be temporary is a motivational factor, once the stories 
change frequently. These data suggest that, in addition to publish their stories, the participants are also interested in interacting socially through this kind of platform. Thus, we asked them: (1) which sociability features of Instagram Stories they knew and (2) how often they use each known feature. The graph in Figure 7 summarizes the obtained results.

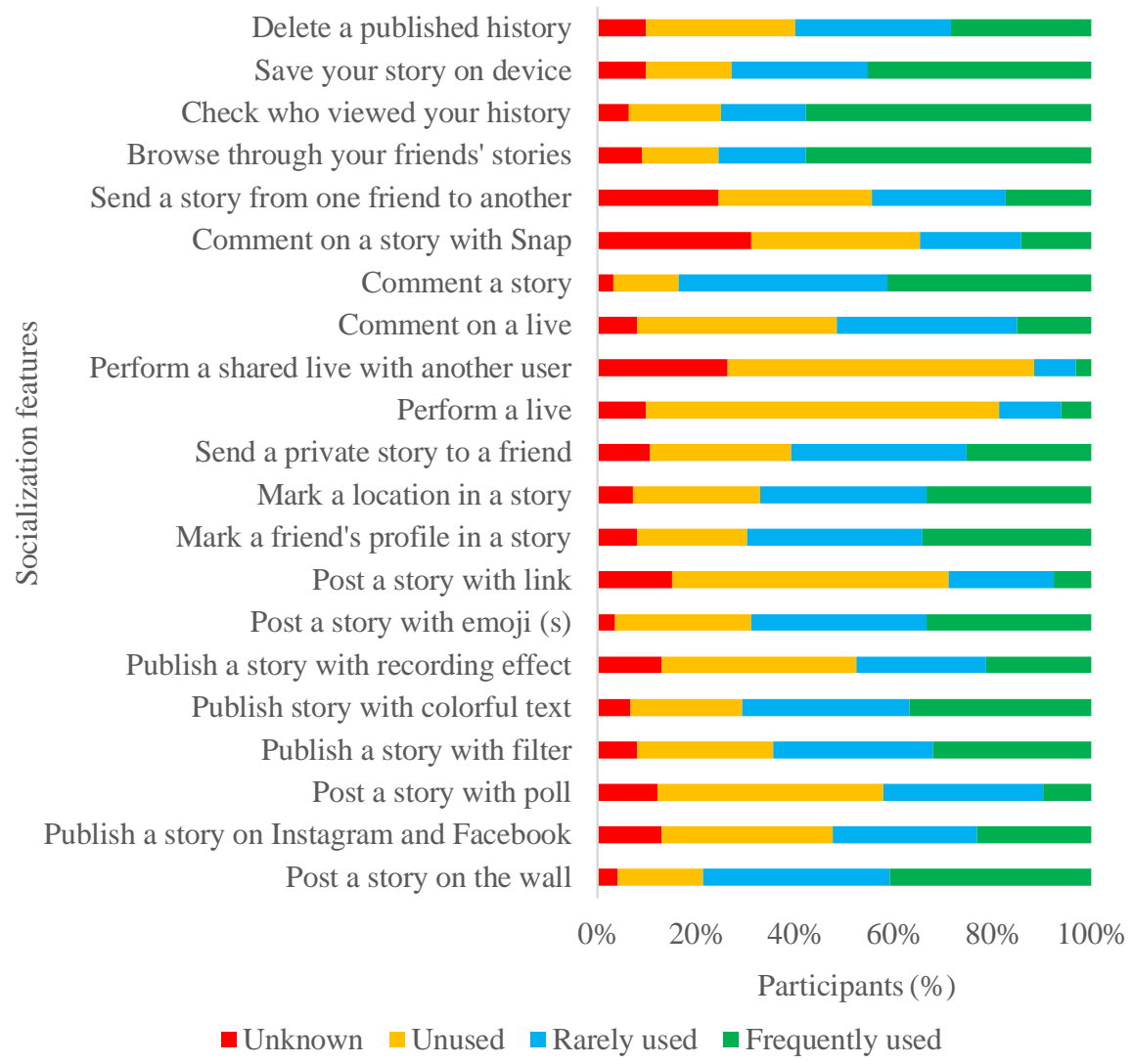

Figure 7. Sociability features of Instagram Stories used by participants

In Figure 7, it is possible to observe that at least $42 \%$ of participants indicated that they did not know, did not use or rarely use all offered features by Instagram Stories to support the sociability among its members. The limited knowledge and use are a warning to interface designers, since more than $60 \%$ of participants make limited use of features that should be used to promote sociability (e.g., (1) comment a story with a snap, (2) perform a shared live with another user and (3) send a published story from one friend to another).

After, we asked participants if the features offered by Instagram Stories have the need for improvements in order to improve their experience and sociability. As a result, the majority $(64 \%)$ of the interviewed answered that some improvements should be implemented on the interface and interaction model to obtain a better social interaction. Complementarily, we used thematic analysis (Braun \& Clarke, 2006) to identify and categorize the suggestions across the participants answers. The graph in Figure 8 summarizes the obtained results. 


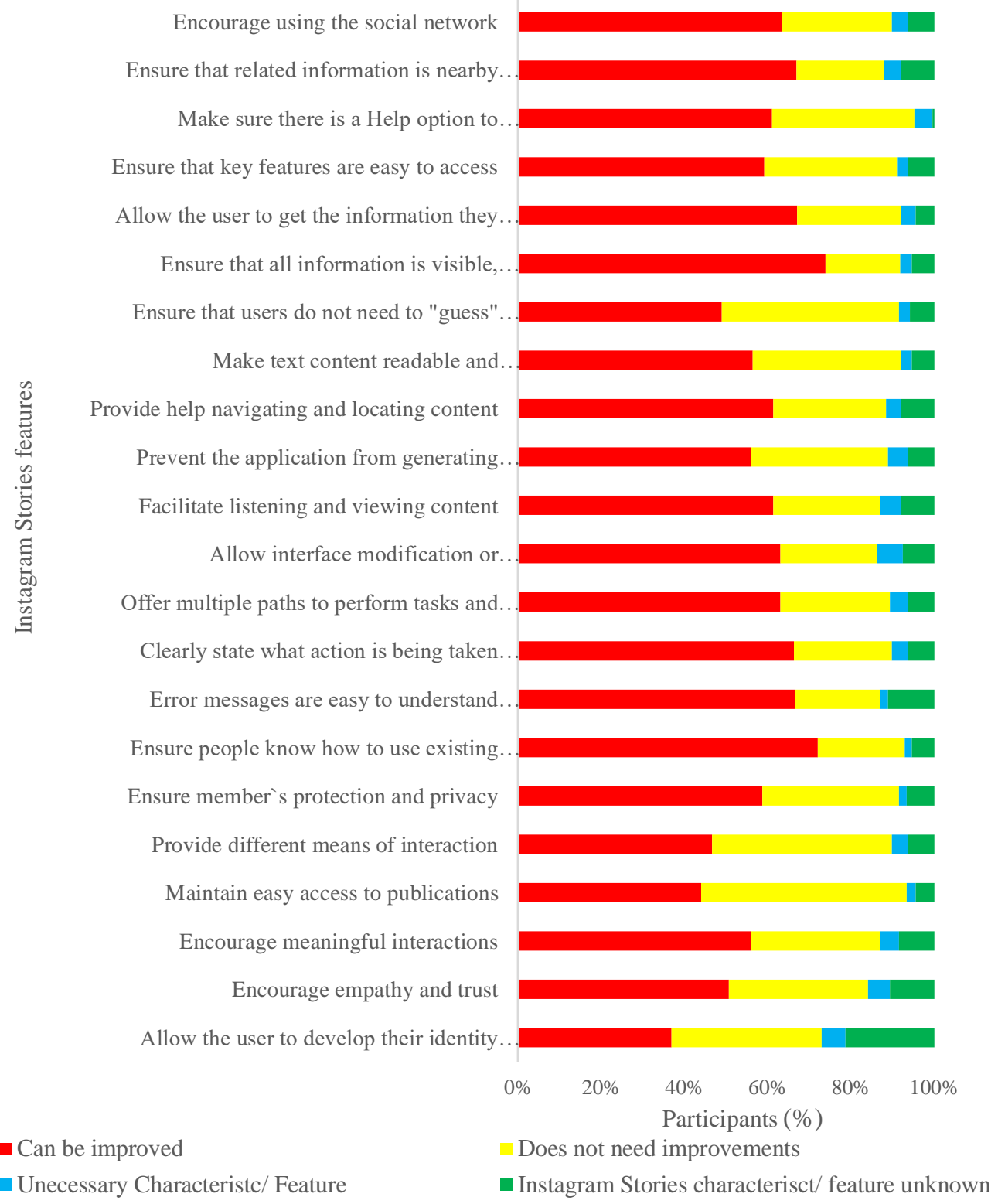

Figure 8. Sociability features of Instagram Stories that, in the users' view point, can be improved

As shown in Figure 8, all participants contributed with suggestions. Among the demands for improvements most cited, by at least $63 \%$ of participants, it is possible to highlight: (1) "Ensure that all information is visible, readable and with easy access" (suggested by $80 \%$ ); (2) "Ensure people know how to use existing functionalities" (suggested by 72\%); (3) "Allow the user to obtain the information they need easily, without needing for memorization" 
(suggested by 67\%) and (5) "Ensure using the social network" (suggested by 63\%). We also observed that most participants' suggestions are related to the (1) ease of use, (2) feedback, (3) flexibility and (4) stimulus of social interaction in different ways.

Thus, these findings reinforce the arguments that Instagram Stories offers sociability features to promote social interaction among its members, but the users may be making a limited use of these features. The limited use and even ignorance of these resources may be justified by the UX and sociability problems exemplified in the previous evaluations.

\section{DISCUSSION OF RESULTS}

In this research, we investigated the following research question: "Does the interface and interaction model of Instagram Stories supports the social interaction among its users?". To answer this question, we evaluated the Instagram stories in perspective of HCI experts and in the users' view point.

Initially, our findings showed that Instagram Stories offers resources to social interaction mediated by the ephemeral communication, such as (1) sharing and visualization of temporary contents and (2) diversified interaction with friends through shared ephemeral contents. However, the HCI experts' evaluation showed that the Instagram's ephemerality proposal violates some guidelines of UX and sociability which aim to stimulates continuous social interaction in this kind of collaborative system. By violating these guidelines, the system may not adequately support the social interaction through the ephemeral communication service (De Souza \& Preece, 2004) (Preece et al, 2015) (Nielsen \& Budiu, 2015) (Bayer et al, 2016).

Later, we showed that, indeed, Instagram Stories' users may be facing difficulties to recognize and to use all offered features to social interaction from ephemeral communication on this system. For a significant number of participants, this system does not use interface and interaction elements consistent with their expectation and experiences. Consequently, Instagram Stories' users may be making limited use of offered features to promote sociability through this service of ephemeral communication. Knowing these limitations, the participants of this study and HCI experts suggested improvements concerning the ease of use, feedback, flexibility and stimulus of social interaction in different ways to improve the experience of use and sociability on Instagram Stories.

These findings evidence the importance of addressing the principles (e.g., guidelines) of UX and sociability on ephemeral communication systems to stimulate the satisfactory experience of use and high sociability. Once the violation of these use qualities may impact in the quality of social interaction mediated by ephemeral communication technologies. In addition, even though the Stories functionality was added in porpoise to stimulate social interaction, the interface and interaction model of Instagram Stories needs to pay more attention in clarify (1) the meaning of its interface elements and (2) the interaction paths, in order to not compromise the social network features' use, once the interface use extensively minimalist elements, but offer them in a satisfactory way. 


\section{CONCLUSION AND FUTURE WORKS}

This work aimed to characterize how Instagram Stories supports the sociability among its members and to discuss the impact of this proposed sociability in the social interaction of users. Taken together, the findings advance our understandings about UX and sociability on collaborative systems of ephemeral narratives. Specifically, this study indicates how the interface and interaction model of Instagram Stories supports and limits the sociability among its users.

This research contributes to Collaborative Systems community and HCI community may reflect about the importance of addressing the principles of UX and sociability in their ephemeral narratives systems. This study also reinforces the importance of sociability as a strategy of use quality to improve user experience and social interaction on collaborative systems.

Furthermore, the adopted methodology might be reproduced in the evaluation of other ephemeral narratives platforms, considering the UX and social interaction of different interest groups (e.g., children, people with disabilities). Therefore, our findings provide insights that are useful for designing of ephemeral communication systems more sociability-focused in the future.

We expect to see continued efforts to advance the findings presented in this study. Thus, as future works we suggest new researches to investigate the sociability through user experience on ephemeral communication systems for different users' groups (e.g., elderly groups, teenagers or people with disabilities) and cultures.

\section{REFERENCES}

Bayer, J.B. et al, 2016. Sharing the small moments: ephemeral social interaction on Snapchat. Information, Communication \& Society, 19(7), pp.956-977.

Braun, V. and Clarke, V., 2006. Using thematic analysis in psychology. Qualitative research in psychology, 3(2), pp.77-101.

Cavalcanti, L.H. et al, 2017. Media, Meaning, and Context Loss in Ephemeral Communication Platforms: A Qualitative Investigation of Snapchat. ACM.

comScore. 2015. Brazil Digital Future in Focus. Available in: 〈https://goo.gl/H51ep1〉. Last access: April $-11,2018$.

De Souza, C.S. and Preece, J., 2004. A framework for analyzing and understanding online communities. Interacting with computers, 16(3), pp.579-610.

Hassenzahl, M., 2013. User experience and experience design. The Encyclopedia of Human-Computer Interaction

Instagram. 2016. Introducing Instagram Stories. Available at: https://goo.gl/nmpvYf. Last access: $11 / 15 / 2017$

Marshall, M.N., 1996. Sampling for qualitative research. Family practice, 13(6), pp.522-526.

Neiva, H. et al. 2016. Diretrizes de UX para Avaliação do Modelo de Interface e Interação de Aplicativos Móveis. In Proc. of SMSI. Belo Horizonte, Brazil.

Nielsen, J., 1994, April. Usability inspection methods. In Conference companion on Human factors in computing systems. ACM. pp. 413-414. 
Nielsen, J. and Budiu, R. 2015. User Experience for Mobile Applications and Websites. In Design Guidelines. 3a Ed., 506 pages.

Preece, J., 2000. Online communities: designing usability, supporting sociability. Industrial Management \& Data Systems, 100(9), pp.459-460.

Preece, J. et al. 2015. Interaction design: beyond human-computer interaction. John Wiley \& Sons.

Statista. 2018. Most famous social network sites worldwide as of January 2018, ranked by number of active users. Available at: https://goo.gl/HtwdDX. Last Access: 11/15/2018.

Wilson, C. et al. 2009. User interactions in social networks and their implications. In Proc. of the 4th ACM European conference on Computer systems. ACM. Nuremberg, Germany, pp. 205-218.

$\mathrm{Xu}$, B. et al. 2016. Automatic archiving versus default deletion: what Snapchat tells us about ephemerality in design. In Proc. of the 19th ACM conference on computer-supported cooperative work \& social computing. ACM. San Francisco, USA, pp. 1662-1675. 